2) Bei Bereitung der gekochten Oele bediene ich mich schon mehrerer Jahre eines blechernen geradrandigen Verdrängungstrichters, und kann ich dieses Verfahren nur empfehlen.

Das Verfahren ist folgendes: 4 Unzen Bilsenkraut u. s. w. werden gröblich zerstossen, mit 3 Unzen Weingeist von $85^{\circ} \mathrm{Tr}$. befeuchtet, und einige Stunden macerirt ; dann wird das feuchte Pulver ohne Druck in den mit Baumwolle verstopften Trichter gethan, und nach und nach mit 32 Unzen Olivenöl übergegossen. Nachdem alles Oel abgetropft ist, wird dasselbe zur Verfliuchtigung des Weingeistes gelinde erhitzt, und noch so viel Oel zugesetzt, dass diess ganze Quantum Oel wieder 32 Unzen beträgt.

Die so bereiteten Oele haben den specifischen Geruch des verwendeten Krauts und die entsprechende Farbe in hohem Grade und sind bei einiger Vorsicht immer klar.

\title{
Ueber Myrrha und Unterscheidung derselben von Bdellium;
}

\section{von \\ Dr. L. F. Bley und E. Diesel.}

Vergleichende Versuche über den Gehalt der Myrrha an ätherischem Oel zeigten eine sehr abweichende Ausbeute. Es wurde natürlich immer Myrrha electa dazu gewählt; der Gehalt an ätherischem Oel wechselte jedoch zwischen 1,6 bis 3,10 Proc. Man beachtete dabei eine sebr sorgfältige und schnelle Abscheidung, da bekanntlich das ätherische Oel sehr geneigt ist, Sauerstoff aufzunehmen, dabei specifisch schwerer wird, sich an die Gefässe als firnissähnliche Masse anhängt, und sich so der Bestimmung entzieht. Wir finden nun, dass die an ätherischem Oel arme Myrrha immer eine starke saure Reaction zeigt, was bei einer guten an ätherischem Oel reichen Myrrha nie gefunden wird. Man sieht hieraus deutlich, dass die erwähnte saure Reaction (welche besonders häufig bei der gewöhnlichen Myrrha vorkommt), abzuleiten ist von einer Oxydation des ätheri- 
schen Oels derselben. und es ist daher eine sorgfältige Aufbewahrung der Nyrrha höhst nothwendig. Feuchtigkeit begünstigt die Oxydation besonders, und man muss also das Befeuchten der Myrrha mit Alkohol, wie es häufig von den Droguisten geschieht, um der bestäubten Myrrha (wie sie nur vorkommen sollte) ein glänzendes, überhaupt besseres Ansehen zu geben, als höchst nachtheilig ansehen.

Bei Bereitung des ätherischen Oels findel man in dem von dem ätherischen Oel befreiten Myrrhenwasser eine starke saure Reaction. Dieses saure Wasser wurde concentrirt, mit etwas kohlensaurem Kalk versetzt, bis keine saure Reaction mehr zu sehen war, die filtrirte Flüssigkeit mil essigsaurem Bleioxyd vermischt, abgedampft und im concentrirten Zustande Alkohol hinzugefügt, wodurch ameisensaures Bleioxyd gefallı wurde. Man zersetzte nun den mit Alkohol gewaschenen Niederschlag mit verdünnter Phosphorsäure, indem man eine Destillation veranstaltete. Das erhattene saure Destillat, mil Alkalien gesättigt, wirkte reducirend auf salpetersaures Quecksilberoxyd und Oxydul, so wie auch auf salpeters. Silberoxyd. Das Verhalten gegen Sublimatlösung und gegen eine verdünte Eisenchloridlösung bewiesen nun nebst den andern Reactionen deutlich das Vorhandensein von Ameisensäure.

Wird das ätherische Myrrhenöl mit Wasser in Beruhrung gesetzt und eine Zeillang Sauerstoff in die Fliissigkeit geleitet, so entsteht allmälig eine saure Reaclion, welche immer mehr und mehr zunimmt, wobei sich das Oel verdickt und zuletzl als eine terpentinartige Masse an das Gefass ansetzt. Dieselbe Erscheinung findet statt, wenn das Myrrhenöl mit Wasser in Berührung längere Zeit der atmosphärischen Luft ausgesetzt wird. Die Producte dieser Oxydation sind Ameisensäure und Weichharz (Balsamharz.)

Dieses Harz löst sich leicht in Aether, ist weich, hat anfangs einen unmerklichen, hinterher einen stark bittern Geschmack, und schmilzt in der Wärme. Es löst sich auch in Alkohol und Terpentinöl. Eine Elementar-Analyse von dem Myrrhenöl, welche noch nicht gemacht zu sein scheint, wäre

Arch. d. Pharm. XCIII. Bds. 3. Hft. 
wohl nothwendig. Jedenfalls ist es ein Kohlenwasserstoff, dem Terpentinöl ähnlich zusammengesetzt Nach der Analyse von Brandes (Almanach fur Scheideliüstler. 1819. S.51 und Berliner Jahrbuch. XXII. S. 275) soll Benzoesäure, sowohl frei als gebunden in der Myrrha vorkommen; es scheinen aber die Reactionen gegen Eisenoxydsalz u s. w. verkannt zu sein, und die freie Säure, welche Brandes für Benzoesäure hielt, ist nichts als Ameisensäure.

Was die Unterscheidung der ächten Myrrha von dem Bdellium und der Pseudo - Myrrha betrifft, so hat neulichst Martiny in Schlitz Versuche angestellt (Jahrbuch fur praktische Pharmacie. B.X. Heft 1. S.25), deren Resultale mit den unsrigen sehr ibereinstimmen. Martiny bemerkt, dass durch Jobs $\mathrm{L}$ in Stuttgart seil meheren Jahren eine sogenannte Myrrha in baculis, welche fruher und später häufig unter der ächten Myrrha gefunden wurde, in den Handel gebracht worden sei. Bei angestellten Versuchen ergab sich, dass diese Pseudo-Myrrha identisch ist mit dem sogenannten Myrrhoid ron P la nche (im Journ. de Pharmac. Août 1810. p. 501. - Jahrb. der Pharmac. IV. 47 beschrieben). Auch Martius scheint sie (das Neueste aus dem Gebiete der Pharmalkognosie. S. 401) beobachtet zu haben. Er sagt, dass sie ein gelbliches, durchsichtiges, wachsglänzendes Gummiharz u. s. W. sei.

Die Pseudo - Myrrha besteht aus verschicden gestalteten durchgehends sehr grossen Stucken, wovon die meisten nur Bruchstiicke eines cylinderformigen Körpers sind. Aeusserlich findet man unregelmässige Erhöhungen und Ver tiefungen, welche von dem Uebereinanderfliessen des hervorquellenden Saftes herzurühren scheinen. Sie besitzt eine matte, graulich bestäubte, undurchsichtige und schmutzig-röthlichbraune Aussenseite. Die Fläche des Bruchs erscheint ziemlich glatt, glasglänzend, dunkelgelb oder bräunlichgelb, fast so durchsichtig wie Senegalgummi. Der Geruch ist schwach myrrhenartig, Geschmack ebenso, hin terher sehr unangenehm bitter, etwas balsamisch, nur wenig kratzend. Salpetersäure lösı die Pseudo-Myrrha zu einer hellgelblichen Fliissigkeit, aus welcher durch Was- 
ser kleine gelbe Pinclchen ausgeschieden werden. Aechte Myrrha wird von Salpetersäure zu einer schleimigen, undurchsichligen, gelblichen oder schmutzig - gelblichen Fluissigkeit mit Hinterlassung eines bräunlichen Bodensatzes aufgelöst; Wasserzusalz bewirkt eine gelbe oder schmutziggelbe Trübung. Bdellium indicum wird von Salpetersäure nicht aufgelöst, es wird weisslich, undurchsichtig und erweicht; Wasser bewirk keine Veränderung in der Flüssigkeit.

M a $\mathrm{l}$ in y fand die Angabe von B on astre, dass Salpetersäure ein sicheres Reagens für Myrrha sei, bestätigt, obschon er nicht jene violette Färbung der Myrrha in Substanz durch Salpetersäure beobachten konnte. Sehr gut eignet sich Fliesspapier, welches mit einem weingeistigen Auszuge von Myrrha befeuchtet wird, bei Zusatz von etwas Salpetersäure, zur Hervorbringung jener blaurothen Färbung. Die Bdelliumsorten, sowie auch die falsche Myrrha, zeigen nur eine gelbe oder bräunliche Farbung.

Das Bdellium indicum unterscheidet sich ferner durch seine gruinlichbraune Farbe, durch schwach myrrhenartigen, mehr terpentinarligen Geruch (der Geruch ist öfters mehr mastix - als myrhonähnlich) und einen bitterlichen. etwas scharfen Geschmack. Abgeschnittene kleine Stiuckchen haben eine undurchsichtige, gelblichweisse oder gelbliche, etwas schmutzige Farbe.

Charakteristisch ist auch das Kleben des Bdelliums, wenn es eine Zeitlang zwischen den Fingern gehalten wird, und der ekelhaft bittere, nicht gewürzhafte Geschmack. Nach dem Alter des Bdelliums scheint der Geruch sehr verschiedenartig zu sein. Eine scheinbar frischere Sorte Bdellium indicum zeigte einen starken myrrhenartigen, zugleich fenchelartigen Geruch.

Die Myrrha electa giebt eine hellgoldgelbe Tinctur und hinterlässt einen undurchsichtigen weisslichen Rückstand.

Die Pseudo - Myrrha yiebt eine hellweingelbe Tinctur und halbdurchsichtigen. weisslichgelben Ruckstand.

Myrrha indica giebt eine dunkelgelbe, etwas ins Orange 
gehende Tinctur, und hinterlässt einen undurchsichtigen weisslichen Ruickstand.

Wasserzusatz giebt bei Myrrha electa eine bläulichweisse milchige Flüssigkeit, bei Myrrha indica weisse milchige Flüssigkeit; die Pseudo - Myrrha giebt keine Veränderung.

Salpetersäure (6 Tropfen zu 20 Tropfen Tinctur) giebt mit Myrrha electa eine gelblichweisse Tribung und Ausscheidung von gelben Harzpuncten.

Nach einiger Zeit färbt sich die Perjpherie der Flüssigkeit, besonders beim Verdunsten des Weingeistes, hellviolett, während im Centrum Niederschlag und Fluissigkeit gelb bleiben. Myrrha indica in demselben Verhältniss (als Tinctur) zu Salpetersäure, wie bei Myrrha electa erwähnt, verhält sich ebenso, nur ist die Trübung dunkler, auch die Färbung der Peripherie und des Centrums dunkler und stärker.

Die Pseudo - Myrrha zeigt keine Veränderung. Beim Erwärmen des Auszuges tritt eine milchige Trübung ein.

Rauchende Salpetersäure (6 Tropfen zu 20 Tropfen Tinctur) giebt mit der Tinctur der Myrrha electa eine umbrabraune, nicht trübe Färbung und Ausscheidung ebenso gefärbter Harzpuncte; nach und nach färbt sich die Flüssigkeit rothbraun, geht ins Violette über und wird beim Verdunsten des Weingeistes an den Rändern des Schälchens schön dunkelviolett. Abgedampft, hinterbleibt ein dunkelguttigelber Rückstand.

Myrrha indica verhält sich gegen rauchende Salpetersäure fast ganz wie Myrrha electa.

Pseudo-Myrrha erleidet durch rauchende Salpetersäure in dem oben erwähnten Verhältnisse der Tinctur keine Veränderung, sie bewirkt beim Erwärmen keine milchige Trubung.

Die Bdelliumsorten, Bdellium indicum und african., unterscheiden sich nur hauptsächlich dadurch, dass die Tincluren derselben weder durch gewöhnliche Salpetersäure, noch durch rauchende, auf irgend eine Art und Weise, eine violette Färbung erleiden.

Wird Myrrha mit destillirtem Wasser geschiittelt (ohngefähr 10 Gran mit 1 Unze Wasser), die Lösung filtrirt, so 
erhält man durch Bleioxydsalze stets einen sarken Niederschlag.

Eine wässerige Lösung, von zwei verschiedenen Sorten Bdellium, als Bdellium indicum vorgekommen, gaben in demselben Verbältniss, von ungefähr 10 Gran Bdellium mit 1 Unze Wasser, wie bei Myrrha angegeben, mit Bleioxydsalzen kaum eine Trübung in der filtrirten Lösung.

Es möchte also auch diese Reaction zur Unterscheidung der Myrrha vom Bdellium zu beachten sein*).

\section{Veber Extractum antiphthisicum, nebst einigen Andeutungen über die Zersetzung der Gerbsäure; \\ Ernst $\stackrel{\text { von }}{\text { Di e s e l. }}$.}

Da dieses Extract auch in Bernburg schon in Anwendung gekommen ist, und daher ofters bereilet wurde, so erlaube ich mir zu der Mittheilung der Herren Weber und Ret$\mathrm{s}$ chy (dies. Archiv B. 39. H. 2.) noch Etwas über die Bereitung dieses Arzneistoffes hinzuzufügen.

Dieses Extract muss natuirlich je nach der Dauer der Berührungszeit der Kalbfelle mit dem Eichenabsud, und überbaupt nach dem Alter der Lohbrihe sehr verschiedenartig zusammengesetzt sein, und muss daher eine ebenso verschiedene Wirkung äussern. - Die Annahme, dass die Lohbrihe eine gute Wirkung, bosonders bei Phthisis habe, wird durch die Erfahrung des Hrn. Dr. W eber bestätigt. Elliatson will sogar die Dämpfe der Lohbrühe gegen Lungensucht, vorziiglich bei Bronchitis chron., wo geringe Entzuindung, grosse Reizbarkeit und viel Secretum sich zeigten, mit gutem Erfolg angewendet haben, indem er die heissen Dämpfe derselben direct auf das kranke Organ einwirken liess. - Meiner Meinung nach kann bloss die sogenannte süsse Lauge zur Bereitung des Extracts gebraucht werden, wesshalb sehr darauf gesehen werden

*) Diese kleine Arbeit wurde im Sommer 1844 unternommen und lag seit dem Herbste desselben Jahrs zum Abdrucke bereit, der indess wegen noch vorhandenen ältern Manuscripts nicht eher bewirkt werden lsonnte,

Die $\mathrm{Ked}$. 